\title{
Tuberculosis detection in nonhuman primates is enhanced by use of testing algorithms that include an interferon $-\gamma$ release assay
}

\author{
JoAnn L. Yee, BS ${ }^{1 *}$; Kamm Prongay, DVM ${ }^{2}$; Koen K. A. Van Rompay, DVM, PhD ${ }^{1,3}$; Suthirote Meesawat, MSc ${ }^{4}$; \\ Taratorn Kemthong, DVM ${ }^{5}$; Bryson Halley, BS ${ }^{1}$; Amanda Carpenter, BS ${ }^{1}$; Peter Nham, MS $^{1}$; Kenneth Rogers, PhD $^{6}$; \\ Dana Hasselschwert, DVM ${ }^{6}$; Francois Villinger, DVM ${ }^{6}$; Alexandra N. Jay, DVM ${ }^{7}$; Saradee Warit, $\mathrm{PhD}^{8}$; \\ Suchinda Malivijitnond, $\mathrm{PhD}^{4,5}$; Jeffrey A. Roberts, DVM ${ }^{1,9}$ \\ 'California National Primate Research Center, University of California-Davis, Davis, CA \\ ${ }^{2}$ Oregon National Primate Research Center, Oregon Health Sciences University, Beaverton, OR \\ ${ }^{3}$ Department of Pathology, Microbiology, and Immunology, School of Veterinary Medicine, University of California-Davis, Davis, CA \\ ${ }^{4}$ Department of Biology, Faculty of Science, Chulalongkorn University, Bangkok, Thailand \\ ${ }^{5}$ National Primate Research Center of Thailand, Chulalongkorn University, Saraburi, Thailand \\ ${ }^{6} \mathrm{New}$ lberia Research Center, University of Louisiana at Lafayette, New Iberia, LA \\ ${ }^{7}$ Veterinary Medicine Division, US Army Medical Research Institute of Infectious Diseases, Fort Detrick, MD \\ 8 Industrial Tuberculosis Team, IMBG, National Center for Genetic Engineering and Biotechnology (BIOTEC), National Science and Technology \\ Development Agency, Pathum Thani, Thailand \\ ${ }^{9}$ Department of Medicine and Epidemiology, School of Veterinary Medicine, University of California-Davis, Davis, CA \\ *Corresponding author: JoAnn Yee (joyee@ucdavis.edu)
}

\section{OBJECTIVE}

To develop a testing algorithm that incorporates multiple assays to evaluate host cellular and humoral immunity and antigen detection concerning Mycobacterium tuberculosis complex (MTBC) infection in captive nonhuman primates.

\section{ANIMALS}

Cohorts of captive-bred and wild-caught macaques from 5 different geographic regions.

\section{PROCEDURES}

Macaques were tested for MTBC infection by use of a $\gamma$ interferon tuberculosis (GIFT) assay, an interferon- $\gamma$ release assay, and other assays. In the first 2 cohorts ( $\mathrm{n}=15$ and I8I), initial validation of the GIFT assay was performed by use of experimentally infected and unexposed control macaques. In the next 3 cohorts $(n=59,42$, and II), results were obtained for opportunistically collected samples from macaques exposed during spontaneous outbreaks.

\section{RESULTS}

Sensitivity and specificity of the GIFT assay in the control cohorts were $100 \%$ and $97 \%$, respectively, and were variable but enhanced by incorporating results from multiple assays in spontaneous outbreaks.

\section{CLINICAL RELEVANCE}

The detection and management of MTBC infection in captive nonhuman primate populations is an ongoing challenge, especially with animal imports and transfers. Despite standardized practices of initial quarantine with regular intradermal tuberculin skin testing, spontaneous outbreaks continue to be reported. Since infection encompasses a range of disease manifestations over time, a testing algorithm that incorporates multiple assays, such as the GIFT assay, to evaluate host cellular and humoral immunity in addition to agent detection is needed. Testing a combination of samples from controlled studies and spontaneous outbreaks of MTBC infection in nonhuman primates would advance the development and validation of a functional algorithm that incorporates promising tools such as the GIFT assay.

$\mathrm{T}$ he detection and elimination of Mycobacterium tuberculosis complex (MTBC) infection is an ongoing problem in the management of nonhuman primate (NHP) colonies in captivity, including research centers and public and private zoos. It is also a concern in the management of many wild NHP populations where tuberculosis (TB) represents a risk to endangered species. The tuberculin skin test (TST) remains the primary tool for diagnosis and surveillance. Originally designed in the early 1900 s to detect active TB in humans, the TST was adapted for use in NHPs almost 50 years ago. It continues to be used, despite documented evidence of its limitations, including unreliable identification of animals with latent MTBC. ${ }^{1}$ The use of the TST has also been impacted by problems in production and quality control of mammalian old tuberculin. ${ }^{1}$ This has led to periodic interruptions in supply, disrupting both routine colony management procedures as well as operation of US CDC quarantine. ${ }^{2}$ 
A series of negative TSTs continues to be required by the CDC to clear import quarantine of NHPs. However, there are multiple documented cases of animals successfully clearing quarantine and then later developing clinical disease after shipment to other facilities, often after having been enrolled in a research study. $^{3-8}$ Potential exposure to MTBC-infected humans is an inherent risk in shipping, particularly if the strict personal protective equipment guidelines used at most NHP facilities are not strictly adhered to. It can be speculated that the stress of manipulation due to shipping, relocation, research procedures, and other factors activates $\mathrm{TB}$; but the triggers for reactivation remain unknown. ${ }^{9}$ Research by Flynn et $\mathrm{al}^{10}$ has clearly demonstrated that TB is not just biphasic (active or latent) but a continual disease spectrum.

Utility of the TST and other diagnostic tests may also be affected by the species of NHP being tested, potentially further complicating test interpretation. ${ }^{10,11}$ Differences in diagnostic sensitivity for both humoral and cellular immunity in African green monkeys (Chlorocebus aetbiops), cynomolgus macaques (Macaca fascicularis), and rhesus macaques (Macaca mulatta) have been documented. ${ }^{11}$ This broad range of disease manifestations over time suggests that a testing algorithm incorporating complementary assays, rather than a single assay focused predominantly on humoral or cellular immunity, will be needed to provide diagnostic accuracy over the course of exposure, infection, and progression.

The sequencing of the $M$ tuberculosis (MTB) genome in $1998^{12}$ and the continuing occurrence of spontaneous outbreaks despite routine skin testing spurred the development of new assays focused in varying degrees on cellular immunity, humoral immunity, or pathogen detection. In 2001, a spontaneous TB outbreak caused by Mycobacterium bovis in a postquarantine, habituated colony of rhesus and cynomolgus macaques occurred at Stanford University. ${ }^{8}$ In addition to the standard TST, samples from animals involved in this outbreak were tested by use of an in vitro INF $\gamma$ release assay (IGRA) known as Primagam (Thermo Fisher Scientific).

When compared with lesions found at necropsy, sensitivity and specificity of the TST were $84 \%$ and $87 \%$, respectively. By contrast, the Primagam test had useful sensitivity (68\%) and excellent specificity (97\%), compared with the disease status, as determined by the presence or absence of gross and histologic lesions indicative of TB. While the TST and Primagam test each yielded false positive results with different samples, use of both assays in parallel was recommended for maximal overall sensitivity in a TB screening program for both rhesus and cynomolgus macaques. ${ }^{8}$ In addition to the TST and Primagam test, paired (pre- and postoutbreak) sera from 57 nonhuman primates from this colony were tested by ELISA for antibodies to early secretory antigenic target 6 (ESAT-6), a small protein secreted by virulent tubercle bacilli. ${ }^{13}$ Of 25 animals with TB lesions at nec- ropsy, 22 (88\%) had high serum levels of the ESAT6 antibody, which has been shown to increase later in infection than the earlier release of interferon- $\gamma$ (IFN $\gamma$ ). The ESAT-6 antibody was found in 16\% (5/32) of postoutbreak sera from animals in which TB could not be confirmed at necropsy. These and other similar, promising new diagnostic tools were reviewed in a 2008 publication. ${ }^{1}$ That review concluded by recommending the evaluation of a combination of tests to develop an algorithm to improve overall sensitivity and specificity.

A 2011 spontaneous outbreak highlighted the ongoing challenge of implementing such an approach. This outbreak involved 11 of 12 rhesus macaques housed in a single room at the Oregon National Primate Research Center (ONPRC). Eight of the 12 macaques originated in China 2 years previously, 3 were colony bred, and 1 was acquired from another primate center. Eleven of the animals had been housed together in the room for more than 6 months. Following routine TST, 6 animals had a positive score (3 with grade 5 and 3 with grade 4 ), 1 had an indeterminate score (grade 3 ), and 3 had no reaction. The remaining 2 animals did not receive a TST due to a research assignment but were negative on the Primagam test and a rapid lateral flow antibody assay (PrimaTB STAT-PAK; Chembio Diagnostics Inc). ${ }^{1}$

All 12 animals were euthanized, and postmortem examinations were performed. Eleven of the 12 macaques had gross and histologic lesions consistent with MTBC, with 2 animals having only rare lesions. Microbial cultures of tracheobronchial lymph nodes were positive for MTBC in 7 of the 12 animals (National Veterinary Services Laboratory). The genotype profile shared the same spoligotype as a 2004 outbreak at another NPRC. Formalin-fixed tissue submitted for IS6110 PCR assay was positive for 3 animals for MTBC species. All 12 terminal samples were negative on the Primagam test; however, the manufacturer of the assay (Prionics) subsequently notified the laboratories that the conjugate reagent in their Primagam kit had deteriorated, thus invalidating results. Positive or weakly positive results were reported for 2 of 12 animals on both ESAT-6 and PrimaTB STAT-PAK. In addition, 6 of 6 animals tested were weakly reactive by an IGRA in the early stages of development at the California National Primate Research Center (CNPRC), and 5 of 10 tested were positive on an early developmental version of the TB-Plex antibody assay. ${ }^{14}$

None of the assays mentioned above in the 2008 review ${ }^{1}$ have since managed to gain acceptance for routine use; in fact, these assays are no longer commercially available. As evidenced by recent outbreaks in various facilities of which we are aware, the need for improved assays remains. The challenge to developing improved tools for surveillance and diagnosis of TB in NHP populations is access to known positive cases to confirm sensitivity of the different assays. The best opportunity is with experimental studies 
with a defined population and documented infection. While studying these populations is the foundation for assay development, these populations do not necessarily represent the best model for evaluating the types of spontaneous outbreaks seen in animals in quarantine, established research colony animals, or group-housed populations in wildlife management settings. Thus, the development and validation of new diagnostic algorithms must utilize a combination of controlled studies and spontaneous outbreaks to inform assay development and refinement.

From our observations of various case studies and outbreaks, we have postulated that reliable surveillance and diagnosis of MTBC in NHPs will require a testing algorithm with multiple assays to evaluate host cellular immunity, host humoral immunity, and agent detection. In support of developing such an algorithm, the Primate Assay Laboratory at the CNPRC developed the interferon tuberculosis (GIFT) test for use in NHPs. It is similar to the IGRAs widely used in humans and cattle. The initial proof of concept was published as a short communication. ${ }^{15}$

The case studies presented in this paper continue our effort to develop a multiassay algorithm to improve MTBC detection in NHPs. In case study 1 , we began by using samples from rhesus macaques infected as controls to titer inoculum for a TB research study. These samples were used to format and collect preliminary data for an IGRA. In case study 2 , we evaluated routine surveillance samples from 2 long-term MTBC-negative breeding colonies to examine the IGRA's specificity. Finally, in 3 additional case studies, we assessed the IGRA's performance within a diagnostic algorithm. Case study 3 featured a wild-caught population; case study 4 , a colony of captive southern pigtailed macaques ( $\mathrm{Ma}$ caca nemestrina); and case study 5, a colony of captive cynomolgus macaques.

\section{Materials and Methods}

\section{Study cohorts}

This study included samples from macaques in controlled, captive environments with (case study 1 controls) and without (case study 2 controls) TB, as well as from both wild-caught (case study 3) and captive (case studies 4 and 5) macaques in which spontaneous infection was found or suspected (Supplementary Figure SI). All animals were maintained with the approval of each organization's Institutional Animal Care and Use Committee under the standards established by the US Federal Animal Welfare Act and the Guide for Care and Use of Laboratory Animals, the Public Health Service Policy on Humane Care and Use of Laboratory Animals, the AVMA Guidelines for the Euthanasia of Laboratory Animals, and the Animal Welfare Assurance No. A4117-01 from the NIH Office of Laboratory Animal Welfare. Sample collection from wild-caught animals was approved by the Department of the National Parks, Wildlife and Plant Conservation, Thailand and the Animal Care and Use
Committee of the National Primate Research Center of Thailand, Chulalongkorn University (protocol review No. 1423007).

\section{GIFT assay}

The GIFT assay ${ }^{15}$ is an IGRA using heparinized whole blood collected and divided into 4 aliquots stimulated overnight $(16 \pm 4$ hours $)$ at $37{ }^{\circ} \mathrm{C}$ with commercially available bovine or avian purified protein derivative (PPD; Thermo Fisher Scientific) and PBS solution or pokeweed and concanavalin A mitogens (Sigma-Aldrich) as internal negative and positive controls, respectively. The stimulated plasma samples were assayed for IFN $\gamma$ by use of commercially available monkey IFN $\gamma$ ELISA reagents as components or a complete kit (MabTech). The manufacturer claims IFN $\gamma$ specificity for the macaque species included in this study. A valid test required an internal positive mitogen control optical density (OD) reading at least 5 times the internal negative PBS control value OD. No avian or bovine PPD OD values $>0.03$ OD units above the unstimulated PBS negative control value was defined as nonreactive and interpreted as a negative response to MTBC. All other OD patterns were interpreted as reactive and suspicious for MTBC infection. Reactivity to bovine PPD that was $\geq 0.03$ OD units greater than the avian PPD OD value was interpreted as positive for MTBC, and reactivity to avian $P P D$ that was $\geq 0.03$ OD units greater than the bovine PPD OD was interpreted as indeterminate. An indeterminate interpretation indicated that reactivity above background was detected, but it was not TB specific and could suggest early infection or cross-reactivity.

\section{TB-Plex assay}

The TB-Plex assay (Charles River Laboratories) is a commercially available assay that utilizes 7 antigencoupled microbeads for the detection of TB antibodies in NHP serum or plasma. The antigen- or controlcoupled beads are combined to create a multiplex test performed in 96-well microtiter plates. Antigenantibody complexes formed during the test serum incubation are detected by incubation with biotinconjugated goat anti-human IgG followed by phycoerythrin-labeled streptavidin. Final results were read and analyzed using multiplex microbead readers (Luminex Corp)..$^{16,17}$

\section{T-Spot TB assay}

The T-Spot TB assay is an enzyme-linked immunosorbent spot (ELISPOT) assay that detects and quantitates the number of IFN $\gamma$-releasing cells from peripheral blood mononuclear cells stimulated with MTB ESAT-6 and culture filtrate protein-10 peptide pools. Multiscreen 96-well plates were coated with anti-IFN $\gamma$ monoclonal antibody B27 (PharMingen) in PBS solution and incubated overnight at $4{ }^{\circ} \mathrm{C}$. The plates were then washed with RPMI 1640 with $10 \%$ fetal bovine serum (growth medium) 4 times before 
blocking with $100 \mu \mathrm{L}$ of growth medium/well for 1 hour at $37^{\circ} \mathrm{C}$. Next, blocking medium was removed and peptide pools (ESAT-6 and culture filtrate protein-10; $0.5 \mu \mathrm{g} / \mathrm{mL}$ each; BEI Resources) were added at $100 \mu \mathrm{L} /$ well. Each positive control well received $0.2 \mu \mathrm{g}$ of phytohemagglutinin-P, and each negative control well received medium only. Then, $2.5 \mathrm{X}$ $10^{5}$ peripheral blood mononuclear cells isolated by density gradient centrifugation from the pigtailed macaques were added to the test wells. After incubation for 28 hours at $37{ }^{\circ} \mathrm{C}$ and $5 \% \mathrm{CO}_{2}$, the plates were washed 4 times before biotinylated anti-human IFN $\gamma$ monoclonal antibody, clone 7-B6-1m (Mabtech) diluted in PBS Tween-20 (Thermo Fisher Scientific) plus $1 \%$ fetal bovine serum, was added at $50 \mu \mathrm{L} /$ well (final concentration, $1 \mu \mathrm{g} / \mathrm{mL}$ ) and incubated at $37^{\circ} \mathrm{C}$ for 3 hours. The plates were again washed 4 times before streptavidin-alkaline phosphatase conjugate (Mabtech) diluted 1:1,000 in PBS was added at $50 \mu \mathrm{L} /$ well and incubated at $37^{\circ} \mathrm{C}$ for 1 hour. The plates were again washed 4 times, and substrate solution (1Step NBT/BCIP substrate solution; Thermo Fisher Scientific) at $50 \mu \mathrm{L} /$ well was added and incubated in the dark for approximately 10 minutes until spots were clearly visible. Finally, the plates were washed with deionized water to stop the reaction and left to dry before spots were counted by use of an immunospot analyzer (CTL S6 Universal-V analyzer; Cellular Technology Ltd).

\section{Results}

\section{Case study I: experimentally infected rhesus macaques}

This group included samples from 15 Indianorigin rhesus macaques (seven 3-year-old females and eight 3-year-old males) that received intrabronchial MTB (Erdman KO1; BEI Resources). Doses ranged from 2 to $100 \mathrm{CFUs} / \mathrm{mL}$. Venous blood samples for GIFT assay were collected on days $0,14,28,42,56$ $(\mathrm{n}=5), 70$ (3), and 84 (2) after infection. Infection progression was confirmed by MTB-specific T-cell response assays. Computed tomography scans were performed at 2-week intervals postinoculation to quantify pulmonary disease. Animals were euthanized and necropsied at 6 to 10 weeks after challenge for anatomic pathology studies and confirmation of infection. These rhesus macaques were experimentally infected with TB as controls to titrate dosage for a subsequent study. No other experimental manipulations were performed.

The GIFT assays demonstrated reactivity beginning at day 14 , when 8 (53\%) of 15 animals had positive results. Earlier date avian PPD reactivity, interpreted as indeterminate at day 14, changed to bovine PPD reactivity greater than avian PPD reactivity, interpreted as positive, by day 28 . By day 28 , all animals with available samples had positive results (Supplementary Figure S2). When the TB-Plex assay was used, no antibody was detected in these experimen- tally infected animals through day 28, suggesting that antibody reactivity took longer to develop than did PPD reactivity.

\section{Case study 2: captive-breeding colony rhesus macaques}

This group comprised GIFT samples collected for routine surveillance from 181 rhesus macaques of both sexes ranging in age from 0.5 to 25 years and housed outdoors in social groups at the CNPRC or ONPRC. Husbandry and management practices were similar at both centers. Animals were fed a laboratory diet (LabDiet 5000 or 5047 , Ralston Purina; Land O' Lakes Inc) twice daily, offered water ad libitum via automatic watering devices, supplemented with fruits and vegetables, and provided with species-appropriate environmental enrichment, manipulanda, and foraging opportunities. Daily health checks and at least annual TST were performed by trained personnel following standard operating procedures. No spontaneous TB infection was found in this group.

Samples for GIFT assay were collected in parallel with TST at both the California and Oregon locations on 181 animals. No skin test reactors were found. Based on nonexposure history and skin test results, 5 GIFT false positives were identified for an overall specificity of $97.2 \%$ or $97 \%(95 / 98)$ at the ONPRC and $98 \%$ (81/83) at the CNPRC (Supplementary Figure S3).

\section{Case study 3: wild-caught cynomolgus macaques}

This group comprised samples for GIFT assay collected from 59 wild-caught cynomolgus macaques. Many of the animals had been trapped as part of an effort to mitigate human-macaque conflict and had known exposure to human communities with a high prevalence of TB infection. Age and sex data were not collected. These macaques were group housed in outdoor enclosures holding 5 to 13 animals each at the Krabok Koo Wildlife Breeding Center, Tha Takiap District, Chachoengsao Province, eastern Thailand. Samples were collected from both cagemates of monkeys that died from TB approximately 6 months prior and from animals housed in neighboring and more distant enclosures. ${ }^{18}$ Samples for GIFT assay were collected in June 2018. Overall, 48 samples were nonreactive and interpreted as negative; 11 samples were reactive and included 8 positive (bovine PPD greater than avian PPD) and 3 indeterminate (avian PPD greater than or equal to bovine PPD) interpretations. Four of the GIFT-reactive samples were also TST reactive, and the other 7 were not. Seven of the 11 were culture positive at later dates.

Researchers at the National Center for Genetic Engineering and Biotechnology, National Science and Technology Development Agency in Thailand previously tested a subset of samples from the same animals in February 2018 using the IGRA-BioTech-v1 developed by their Industrial Tuberculosis Team and also subsequently in August 2018 using a 
new, higher dilution version of the IGRA-BioTech$\mathrm{v} 2 .{ }^{18}$ The IGRA and TST reactivity for the 11 GIFTreactive samples in June 2018 along with available earlier (February) and later (August) IGRA-BioTech results and parallel TST reactivity from the same animals were summarized (Figure I).

\section{Case study 4 : captive pigtailed macaques}

A spontaneous outbreak in a captive research colony provided an opportunity to test samples from 42 southern pigtailed macaques imported from Indonesia. Histories received with these samples indicated that the animals were from a remaining subset from a recent shipment that comprised 30 females aged 3 to 12 years and 15 males aged 4 to 6 years that had received 3 TSTs over the 31-day quarantine-upon-arrival period. During quarantine, 1 macaque in the shipment had an atypical TST reaction with some swelling noted on days 1 and 2, but the swelling resolved by day 3 without reaching the positive threshold. Nevertheless, samples were submitted to Charles River Research Animal Diagnostic Services for antibody analysis by use of the TB-Plex assay, and all were negative. As a precaution, the shipment group remained quarantined for several additional weeks.

Approximately 10 weeks later, the macaque with the atypical TST result and 1 other animal were euthanized for clinical signs of infection of undetermined origin. Necropsy findings in the animal with the previous atypical TST suggested disseminated TB that was confirmed by PCR assay (National Veterinary Services Laboratory). Liver nodules with no confirmation of TB were found in the second animal. Blood samples from these 2 suspicious animals were not available for testing in this study. Subsequently, another 4 animals were euthanized due to clinical signs of infection. Postmortem findings supporting infection were summarized (Supplementary Table SI).

The remaining 42 animals were tested by TST, PCR assay, and ELISPOT assay on site. Samples from these 42 macaques were also submitted to the Primate Assay Laboratory for GIFT assay. The GIFT assay yielded 13 reactive samples. Seven were interpreted as positive (bovine PPD reactivity greater than avian PPD), and 6 were interpreted as indeterminate (avian PPD reactivity greater than bovine $P P D)$. The remaining 29 were nonreactive and interpreted as negative results (although many of these negative results had high baseline levels of IFN $\gamma$ in unstimulated plasma that could have potentially confounded interpretation). Ten samples were reactive (6 positive and 4 weak positive or indeterminate) in the ELISPOT assay. Overall, 26 samples were nonreactive on both assays and 7 were reactive on both; 6 were reactive on GIFT assay alone, and 3 were reactive on ELISPOT assay alone (Supplementary Figure S4). Results of plasma antibody testing for the samples submitted for GIFT assay were negative using the TB-Plex assay.

\section{Case study 5: captive cynomolgus macaques}

This group comprised samples from 7 male and 4 female captive-bred cynomolgus macaques that were 5 to 11 years of age for which the GIFT assay was performed following incidental findings of gross and histologic lesions indicative of $\mathrm{TB}$ at necropsy and MTBC-positive results of PCR assay (National Veterinary Services Laboratory) in formalin-fixed tissues from another member of their cohort. The histories received with the samples noted that the samples were from a remaining subset of a larger group of over 200 research macaques that included an animal with gross and histologic lesions indicative of MTBC at necropsy, despite no TST-positive reports, for the 2 years they were housed at the institution. Histories indicated that the animals were subjected to 3 to 5

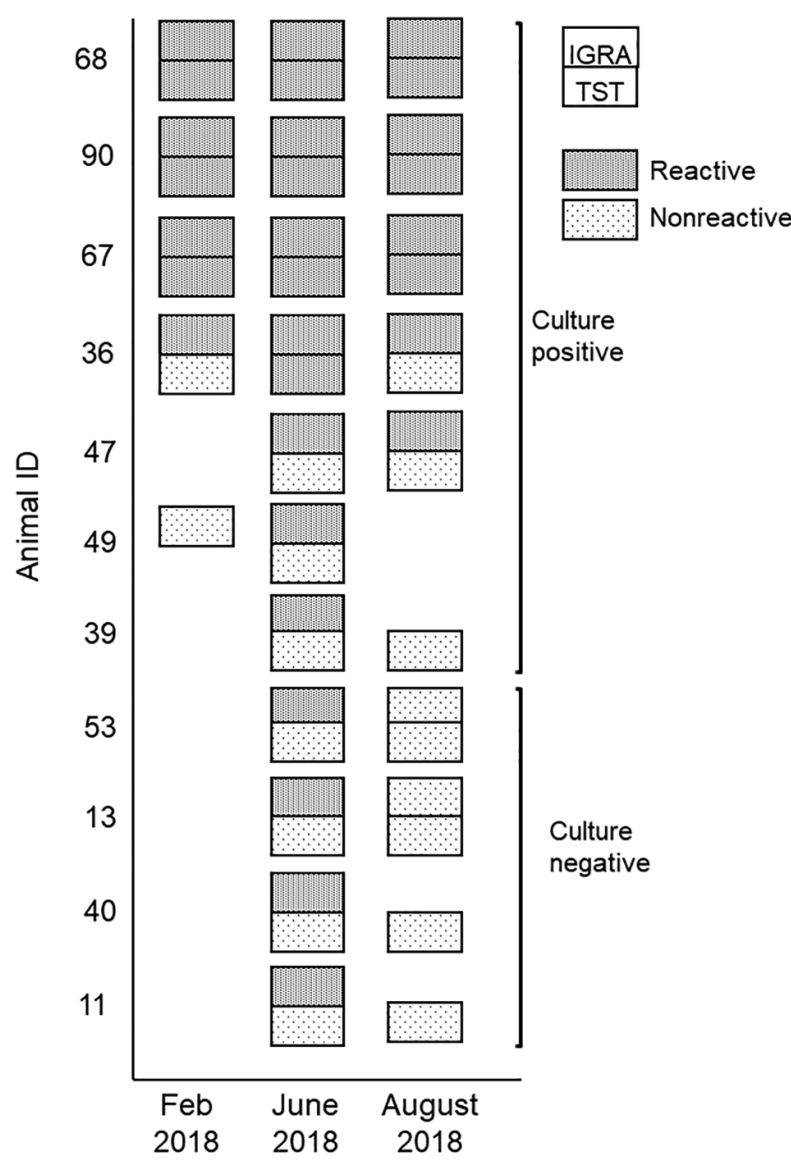

Figure I-Interferon- $\gamma$ release assay (IGRA) and tuberculin skin test (TST) results for detection of Mycobacterium tuberculosis complex (MTBC) infection in the cynomolgus macaques (Macaca fascicularis; $n=59$ ) in case study 3 . The $\gamma$ interferon tuberculosis (GIFT) assay results in June 2018 are compared with available earlier (February 2018) or later (August 2018) IGRA and TST results. The reactive group includes all results interpreted as either positive or indeterminate-weak positive. The nonreactive group includes all results interpreted as negative. As assay development progressed, different variations of the IGRAs were used at each time point: February is the BioTech-vl assay, June is the GIFT assay, and August is the BioTech-v2 assay. Microbial culture results were from samples collected August 2018 or at later dates. 


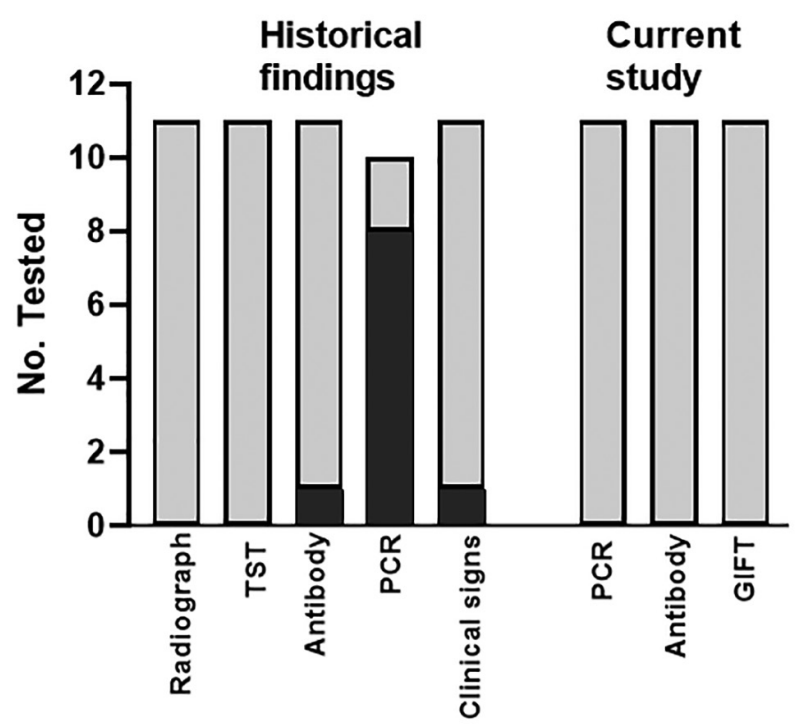

Figure 2-Summary of historical and present study findings for II cynomolgus macaques of case study 5 . The GIFT, PCR, and antibody assays of study samples did not confirm MTBC infection despite prior testing and clinical signs suggestive of infection. The darker bars represent the number of positive and the lighter the number of negative results. See Figure I for remainder of key.

TSTs, 3-view thoracic radiography, MTBC PCR assay, and MTBC serology testing.

No TST reactivity or radiographic evidence was seen in any of the animals (Figure 2). One animal was positive for antibody (Intuitive Biosciences). Bronchoalveolar lavage samples yielded positive results of MTBC PCR assay for 8 animals and negative results for 2 animals; 1 macaque was not tested. However, subsequent repeated PCR testing of all animals yielded negative results. Clinical signs, specifically reports of chronic coughing, were only apparent in 1 macaque that was also antibody reactive, and its sample reacted weakly to avian PPD only. No samples were positive on GIFT assay.

\section{Discussion}

The surveillance of MTBC infection in NHP colonies has been an ongoing challenge since the 1990s. ${ }^{19}$ Shortages of mammalian old tuberculin have disrupted importation of NHPs and prevented ongoing surveillance of MTBC in domestic colonies. The NHP research community needs to utilize the improved tools of 21st century laboratory medicine to develop reliable, validated, adjunct assays to the classic TST to detect MTBC in NHPs.

The current TST only addresses the host cellmediated immunity and is susceptible to errors in administration and interpretation. ${ }^{1}$ IGRAs, similar to the Quantiferon blood test used in humans, are an alternative to the TST for detecting cell-mediated immunity. ${ }^{1}$ Using IGRA principles, we configured the GIFT assay for NHPs. We initially showed that the GIFT assay performed well in 15 experimentally infected rhesus macaques (case 1) as early as 14 days after inoculation.
Although our data were limited and GIFT results remained positive in all samples tested, beyond day 48 postinfection, the level of IFN $\gamma$ released appeared to decrease. This result agreed with reported findings for experimental MTB infection in 8 cynomolgus macaques. ${ }^{20}$ In that study, ${ }^{20}$ animals were evaluated for several parameters over a 6-week period. An IFN $\gamma$ response as measured by ELISPOT assay was detected to some antigens by 2 weeks postinfection and had increased to multiple antigens by 5 to 6 weeks postinfection. The results indicated that as infection progressed, the IFN $\gamma$ responses increased in both magnitude and range of antigens. The authors also reported that this pattern fit with findings of longer-term studies that show the immune response gradually broadens but then is reduced as infection is controlled. ${ }^{20}$ Results for those cynomolgus macaques and the rhesus macaques of case study 1 demonstrated the value and changing sensitivity over time of IFN $\gamma$ assays in detecting MTBC infection.

In contrast to our observations for rhesus macaques with no history of TB infection (case study 2 ), the cases involving infected members had high baseline IFN $\gamma$ values in unstimulated blood from some animals. Further research is needed to determine the significance of these elevated values. If they are high enough to mask an increase to specific MTBC stimulation, they could result in a false negative interpretation; conversely, they might also serve as a positive nonspecific marker of infection. We also observed a higher response to avian PPD in groups with infected members than in uninfected members. More investigation is needed to determine whether these data could also be useful as screening markers. Data from case study 1 suggested that the response to avian PPD is stronger than that to bovine PPD at early time points (day 14), but this response converts to bovine PPD greater than avian PPD by day 28 . Thus, reactivity to avian PPD may be important to consider as a marker of early infection and may also be another area for future study.

When applied to spontaneous outbreak situations described in case studies 3, 4, and 5, the GIFT assay did detect samples from infected animals that were not positive by use of the CDC-mandated skin test in those populations. Unlike the first 2 populations (rhesus macaques), these outbreaks occurred in different macaque species (cynomolgus and southern pigtailed macaques). While the basic pathogenesis of TB infection should be similar, it could affect the timing or strength of the test results. These samples were from populations in which an animal had been characterized as infected on the basis of pathogen detection or necropsy findings. In some but not all cases, positive results of skin tests, antibody assays, or PCR assays were also reported within the population. Unfortunately, samples from infected animals were not always available for inclusion in the test groups. Additional TST or PCR data were available for some but not all animals that had samples included in this study. 
A lack of parallel tests from the same time points precluded a direct comparison of the assays and determination of analytical sensitivity. This is the basic challenge of evaluating surveillance or diagnostic tests in a spontaneous outbreak; when TB-suspect animals are identified in any population of captive or feral animals, the actions of colony managers are not focused on systematic collection of samples but rather on dealing with the practical effects of the outbreak by removing and euthanizing the animal as soon as possible.

As was shown in earlier outbreak reports, ${ }^{8,18}$ data from case 3 animals showed that IGRA and TST results corresponded in some but not all animals and that diagnostic sensitivity could be increased by interpreting reactivity to either the IGRA or TST as indicative for infection. In this population, the IGRA was more sensitive than the TST. Combining the IGRA and ELISPOT reactivity in case study 4 similarly improved sensitivity. Our findings suggested that cellmediated immunity is clearly a useful marker, and 1 or more assays such as the GIFT assay would be an important component of a TB testing algorithm.

Commercially available assays for detection of antibody against MTBC were used as a measure of humoral antibody in case study 1 . None was detected, but we had only limited samples available and most

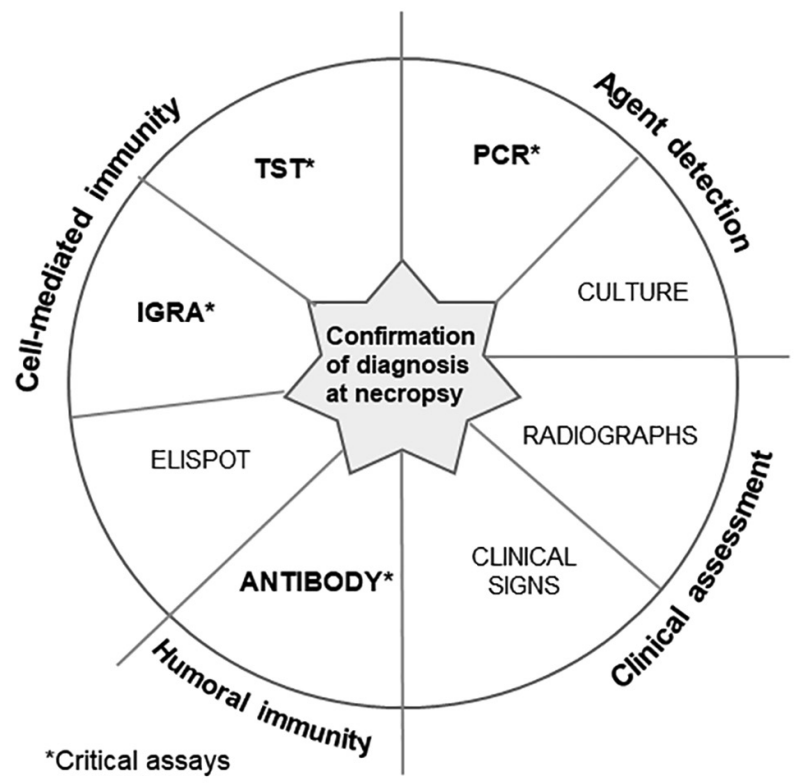

Figure 3-Testing algorithm developed for MTBC infection. Detection of MTBC infection and assessment of cellular and humoral immune responses depend on systematic collection of parallel samples to compare the presence of all 3 parameters beginning from the time of initial exposure and continuing over the time course of infection. This information can be obtained from experimental infections, but tracking the time course of spontaneous outbreaks as they are detected is also critical to interpretation of results. While it is desirable to compare as many assays as possible at baseline, continuing weekly until euthanasia, experimental considerations or the circumstances of a spontaneous outbreak may limit options. Confirmation of infection at necropsy, and recovery of the organism at necropsy if possible, is most critical. were collected within 48 days after experimental TB inoculation. ${ }^{15}$ In general, antibody responses can take weeks to months to develop. These antibody assays rely on patterns of reactivity to a panel of TB antigens. Greater than $99 \%$ specificity has been reported for the TB-Plex assay, and, when used in a spontaneous outbreak among cynomolgus macaques, increasing seropositivity from $2 \%$ at baseline to $8 \%$ at 12 to 14 weeks has been reported. ${ }^{17}$ By use of the TB-Plex reagents, antibody was not detected in the case 4 and case 5 samples. Decreasing cell-mediated and increasing humoral immunity over time suggest that assays for both will be needed in a TB testing algorithm.

For direct agent detection, the PCR assay for MTBC detection is both sensitive and specific; however, it is often difficult to obtain an appropriate antemortem sample. Gastric and bronchoalveolar lavage samples will be negative even in infected animals unless collected during peak bacterial loads. Primer and probe sequences have been published, ${ }^{21}$ and the assay is available from reference laboratories. Although thoracic radiography and acid-fast bacteria smears may not distinguish MTBC organisms from environmental and other mycobacteria, they may also be useful and beneficial in a testing algorithm. Microbial culture has historically been considered the gold standard; however, it is logistically difficult and may lack sensitivity. MTBC organisms grow slowly and may require up to 6 weeks in culture under strict biosafety precautions before detection. Similar to the situation with the PCR assay, appropriate samples may be difficult to obtain. Published studies $9,10,18,20$ have shown that MTBC-infected NHPs are not always culture positive.

To determine the optimal testing algorithm for detection of TB in macaques would require testing large numbers of uninfected and infected animals. The potentially confounding influence of various NHP species and MTBC strains would need to be addressed. The threat posed by TB worldwide has led to an expansion in TB research. The need for a cellular and humoral combined assay in MTBC detection was previously identified in a publication to which one of the authors of this report contributed. ${ }^{1}$ The challenge has always been how to support and conduct such a combined study in an animal biosafety level 3 environment.

One option is that the development and validation of a useful TB algorithm could potentially be piggybacked on another study that involves experimental infection, such as in case 1. Supplemental funding for a study to simultaneously sample a population of known exposed rhesus macaques could provide the material to assess a testing algorithm with multiple assays that address host cellular immunity, host humoral immunity, and agent detection. While it is relatively easy to acquire and test samples from uninfected animals to determine the specificity of each assay, determining sensitivity is much more difficult.

The occurrence of spontaneous MTBC outbreaks could also be employed in algorithm development if a process for standardized collection of samples was de- 
veloped for testing. One possible model is the CDC and state public health laboratory programs providing specific samples to CDC-designated laboratories for testing in suspected outbreaks of specific agents such as Burkbolderia sp or Ebola virus. ${ }^{22}$ Another possible approach would be to utilize groups such as the pathogen detection laboratories present in the NPRC consortium system to serve as reference laboratories by facilities encountering spontaneous outbreaks of TB in their quarantine or colony. Several of these laboratories have historically served as reference laboratories for the screening and confirmation of simian retroviral infections. ${ }^{23}$ By publication on the NPRC websites, the participating laboratories could be contacted to ensure systematic collection of samples for humoral and cellular immune assays as well as samples to detect the MTBC agent. The combination of controlled laboratory studies with an established protocol for sample collection in spontaneous outbreaks will provide data sets that will allow validation of current promising tools for an MTBC testing algorithm. Suggested assays to incorporate in a study to define a testing algorithm are shown (Figure 3 ).

In conclusion, the development of reliable algorithms for the diagnosis of TB is urgently needed and should be based on the following considerations: time course of cellular and humoral immune responses during infection and disease progression, NHP species-specific differences, the source of the animals (captive bred vs wild caught or imported), and validation with data sets from both laboratory and field studies. Only by developing and implementing reliable testing algorithms will we become more successful in excluding and eradicating this serious pathogen from captive and managed wild populations.

\section{Acknowledgments}

Funded in part by the Office of Research Infrastructure Programs/OD (grant Nos. P51 OD011107, 5U42OD010990 and P51 OD011092-60, U42 OD010426-15), the Thailand Research Fund Senior Scholar Grant (No. RTA6280010), and the Thailand Science Research and Innovation Fund given to Suchinda Malivijitnond.

The authors declare that there were no conflicts of interest.

The authors thank the clinicians and researchers who generously shared samples for testing as well as the laboratory and animal care staff at our institutions, including Marcelo Delos Reyes III, Heidi Palmer, and Brittany Sabin, for their project support.

\section{References}

1. Lerche NW, Yee JL, Capuano SV, Flynn JL. New approaches to tuberculosis surveillance in nonhuman primates. ILAR J. 2008;49(2):170-178.

2. Roberts JA, Andrews K. Nonhuman primate quarantine: its evolution and practices. ILAR J. 2008;49(2):145-156.

3. Jay A, Marko S, Luke $\mathrm{K}$, et al. When the tuberculin skin test fails: one institution's experience with the identification of TB in research cynomolgus macaques. Presented at: Association Of Primate Veterinarians 46th Annual Workshop; October 24-27, 2018; Baltimore, MD.

4. Payne KS, Novak JJ, Jongsakul K, et al. Mycobacterium tuberculosis infection in a closed colony of rhesus macaques ( $\mathrm{Ma}$ caca mulatta). J Am Assoc Lab Anim Sci. 2011;50(1):105-108.

5. Obaldía N III, Nuñez M, Montilla S, Otero W, Marin JC. Tu- berculosis (TB) outbreak in a closed Aotus monkey breeding colony: epidemiology, diagnosis and TB screening using antibody and interferon-gamma release testing. Comp Immunol Microbiol Infect Dis. 2018;58:1-10. doi:10.1016/j. cimid.2018.06.007

6. Mätz-Rensing K, Hartmann T, Wendel GM, et al. Outbreak of tuberculosis in a colony of rhesus monkeys (Macaca mulatta) after possible indirect contact with a human TB patient. $J$ Comp Patbol. 2015;153(2-3):81-91.

7. Panarella ML, Bimes RS. A naturally occurring outbreak of tuberculosis in a group of imported cynomolgus monkeys (Macaca fascicularis). J Am Assoc Lab Anim Sci. 2010;49(2):221-225.

8. Garcia MA, Yee J, Bouley DM, et al. Diagnosis of tuberculosis in macaques, using whole-blood in vitro interferon-gamma (PRIMAGAM) testing. Comp Med. 2004;54(1):86-92.

9. Flynn JL, Gideon HP, Mattila JT, Lin PL. Immunology studies in non-human primate models of tuberculosis. Immunol Rev. 2015;264(1):60-73. doi:10.1111/imr.12258

10. Scanga CA, Flynn JL. Modeling tuberculosis in nonhuman primates. Cold Spring Harb Perspect Med. 2014;4(12):a018564. doi:10.1101/cshperspect.a018564

11. Brusasca PN, Peters RL, Motzel SL, Klein HJ, Gennaro ML. Antigen recognition by serum antibodies in non-human primates experimentally infected with Mycobacterium tuberculosis. Comp Med. 2003;53(2):165-172.

12. Cole ST, Brosch R, Parkhill J, et al. Deciphering the biology of Mycobacterium tuberculosis from the complete genome sequence. Nature. 1998;393(6685):537-544.

13. Kanaujia GV, Garcia MA, Bouley DM, Peters R, Gennaro ML. Detection of early secretory antigenic target- 6 antibody for diagnosis of tuberculosis in non-human primates. Comp Med. 2003;53(6):602-606

14. Yee J, Dhawan R, Roberts J. The ongoing search for improved diagnostic tools to detect tuberculosis in nonhuman primates. Abstract presented at: Association of Primate Veterinarians 45th Annual Workshop; October 19-22, 2017; Cedar Creek, TX.

15. Yee JL, Prongay $\mathrm{K}$, Miles $\mathrm{B}$, et al. Interferon gamma test for the detection of Mycobacterium tuberculosis complex infection in Macaca mulatta and other non human primates. $J$ Med Primatol. 2019;48(4):260-263.

16. Serologic Methods Manual: Multiplexed Fluorometric ImmunoAssay (MFIA) for Tuberculosis. Charles River Laboratories; 2017.

17. Dhawan R, Wunderlich M. Detection of TB antibodies in naturally and experimentally infected nonhuman primates using TB-Plex serology assay. Presented at: Association of Primate Veterinarians 47th Annual Workshop; October 9-12, 2019; Broomfield, CO.

18. Warit S, Billamas P, Makhao N, et al. Detection of tuberculosis in cynomolgus macaques (Macaca fascicularis) using a supplementary Monkey Interferon Gamma Releasing Assay (mIGRA). Sci Rep. 2020;10(1):16759. doi:10.1038/s41598-020-73655-3

19. Abee C. Letter from APV President to Synbiotics. APV Newsletter. August 1997.

20. Lin PL, Pawar S, Myers A, et al. Early events in Mycobacterium tuberculosis infection in cynomolgus macaques. Infect Immun. 2006;74(7):3790-3803. doi:10.1128/IAI.00064-06

21. Wolf TM, Mugisha L, Shoyama FM, et al. Noninvasive test for tuberculosis detection among primates. Emerg Infect Dis. 2015;21(3):468-470. doi:10.3201/eid2103.140052

22. Submitting specimens to CDC. CDC. Accessed September 7 , 2021. https://www.cdc.gov/laboratory/specimen-submission/ help-faqs.html

23. Pathogen detection assay group. NPRC. Accessed September 7, 2021. https://www.nprcresearch.org/primate/pathogendetection/pathogen-detection-working-group.php

\section{Supplementary Materials}

Supplementary materials are posted online at the journal website: avmajournals.avma.org 